\title{
Glutamine Imaging: A New Avenue for Glioma Management
}

\author{
(D) S. Ekici, (D).A. Nye, (D).G. Neill, (D).W. Allen, (D).-K. Shu, and (D)C.C. Fleischer
}

\begin{abstract}
SUMMARY: The glutamine pathway is emerging as an important marker of cancer prognosis and a target for new treatments. In gliomas, the most common type of brain tumors, metabolic reprogramming leads to abnormal consumption of glutamine as an energy source, and increased glutamine concentrations are associated with treatment resistance and proliferation. A key challenge in the development of glutamine-based biomarkers and therapies is the limited number of in vivo tools to noninvasively assess local glutamine metabolism and monitor its changes. In this review, we describe the importance of glutamine metabolism in gliomas and review the current landscape of translational and emerging imaging techniques to measure glutamine in the brain. These techniques include MRS, PET, SPECT, and preclinical methods such as fluorescence and mass spectrometry imaging. Finally, we discuss the roadblocks that must be overcome before incorporating glutamine into a personalized approach for glioma management.
\end{abstract}

ABBREVIATIONS: $\mathrm{ASCT} 2=$ alanine, serine, cysteine transporter 2; ${ }^{11} \mathrm{C}-\mathrm{Gln}=5-{ }^{11} \mathrm{C}-(2 \mathrm{~S})$-glutamine; DMI $=$ deuterium metabolic imaging; $\left.{ }^{[8} \mathrm{F}\right]-\mathrm{FGln}=4-\left[{ }^{18} \mathrm{~F}\right]-$ $(2 \mathrm{~S}, 4 \mathrm{R})$-fluoroglutamine; $2-\mathrm{HG}=2$-hydroxyglutarate; $I D H=$ isocitrate dehydrogenase; $\mathrm{MSI}=$ mass spectrometry imaging; $\mathrm{mTOR}=$ mechanistic target of rapamycin; NMR = nuclear MR; PRESS = point-resolved spectroscopy; TCA = tricarboxylic acid

\section{Abnormal Glutamine Metabolism in Gliomas}

G liomas are one of the most common types of brain tumors, and accurate diagnosis relies on imaging and histology. ${ }^{1}$ While histology can identify the tumor grade, isocitrate dehydrogenase (IDH) mutation status, and other pathologic markers, it requires invasive tissue sampling and is impractical for treatment monitoring. The recent emergence of treatments targeting cancer metabolism in clinical trials, combined with poor prognosis particularly for high-grade gliomas, necessitates new noninvasive metabolic imaging strategies capable of stratifying patients, monitoring treatment response, and prognostication. Here, we describe the importance of glutamine in glioma progression and management, followed by a review of imaging techniques capable of quantifying glutamine concentrations.

A hallmark of many cancers, including gliomas, is metabolic reprogramming that enables cancer cells to divide quickly and

Received May 5, 2021; accepted after revision August 4.

From the Departments of Radiology and Imaging Sciences (S.E., J.A.N., J.W.A., C.C.F.), Pathology and Laboratory Medicine (S.G.N.), Radiation Oncology (H.-K.S.), and Neurology (J.W.A.), Emory University School of Medicine, Atlanta, Georgia; and Wallace H. Coulter Department of Biomedical Engineering (C.C.F.), Georgia Institute of Technology and Emory University, Atlanta, Georgia.

Please address correspondence to Candace Fleischer, PhD, 1841 Clifton Rd NE, Rm 290, Atlanta, GA 30029; e-mail: candace.fleischer@emory.edu; @fleischer_lab

- Indicates open access to non-subscribers at www.ajnr.org

http://dx.doi.org/10.3174/ajnr.A7333 evade clearance by remodeling the tumor microenvironment. ${ }^{2}$ One of the most common examples of reprogramming is the Warburg effect, evidenced by high rates of glycolysis and lactate production, even in the presence of oxygen. In addition to high glucose consumption, gliomas also have increased rates of glutamine intake and require larger stores of glutamine for survival compared with healthy cells. ${ }^{3}$ Glutamine is an important energy substrate and carbon source for cancer cells, and glutamine "addiction" is emerging as a hallmark of many cancers. ${ }^{4}$ Glutamine is catabolized to several by-products that aid in cancer proliferation, including glutamate via glutaminolysis and $\alpha$-ketoglutarate as an intermediate of the tricarboxylic acid (TCA) cycle. ${ }^{5}$ Most primary World Health Organization grade II and III infiltrating gliomas and secondary glioblastomas (World Health Organization grade IV) exhibit IDH mutations that produce 2-hydroxyglutarate (2HG) rather than $\alpha$-ketoglutarate. ${ }^{5}$ Glutamine is also a vital nitrogen source for the production of amino acids, nucleotides, fatty acids, and polyamines necessary for proliferation. Cancer cells have an increased demand for nitrogen, and large stores of glutamine enable high rates of synthetic biomass generation and TCA cycle anaplerosis. ${ }^{4}$

The intake of nutrients including glutamine by noncancerous cells is limited by growth factors. In cancer cells, increased consumption of glutamine is, in part, facilitated by oncogenic drivers including Myc and the mechanistic target of rapamycin (mTOR). Myc increases expression of glutamine transporters, modulates expression of glutaminase, and activates enzymes involved in 
purine and pyrimidine synthesis. ${ }^{2,4}$ Myc-induced metabolic reprogramming even precedes $\mathrm{MR}$ imaging-observable tumor growth. ${ }^{6}$ Mice with Myc-activated hepatocytes display increased expression of genes related to glutamine transport and breakdown in the pretumor state, including glutaminase and glutamine dehydrogenase. ${ }^{6}$ Increased mTOR signaling is commonly associated with cancer proliferation and can be activated in cancers with high concentrations of intracellular glutamine mediated through the alanine, serine, cysteine transporter 2 (ASCT2). ${ }^{7}$ mTOR stimulates glutaminolysis in cancer cells by increasing the activity of glutamine dehydrogenase and promoting the breakdown of glutamine into TCA cycle intermediates. ${ }^{4}$

Glutamine use in cancer cells varies within and between tumors due to dynamic interactions among the tumor microenvironment, oncogenes, tissue type, and nutrient availability. ${ }^{5,8}$ Myc-induced differences in glutamine transporters and glutaminase concentrations result in some gliomas accumulating large stores of glutamine, while others readily catabolize glutamine., ${ }^{9,10}$ Gliomas with high rates of glutamine catabolism exhibit metabolic plasticity, resistance to treatment, and a mesenchymal phenotype. ${ }^{11}$ Mesenchymal gliomas in mice have increased glutamine concentrations compared with healthy brain tissue and nonmesenchymal glioma subtypes. ${ }^{12}$ These results have been demonstrated in patients with gliomas using glutamine-based PET, with high glutamine concentrations observed in progressing gliomas compared with the surrounding brain tissue or clinically stable tumors. ${ }^{13}$ Because mesenchymal subtypes are generally associated with worse outcomes, glutamine concentrations may indicate prognosis. Mice treated with chemotherapy show a significant decrease in tumoral glutamine concentrations, supporting the importance of in vivo glutamine quantification after treatment. ${ }^{13}$ Increases in glutamine flux, ie, conversion of glutamine to glutamate, have been observed in glioma-bearing mice treated with temozolomide, a potential explanation for glutamine decreases following chemotherapy. ${ }^{14}$ Because inhibiting glutamine metabolism limits cancer growth and promotes antitumor immunity in the microenvironment, ${ }^{15}$ the glutamine pathway has been identified as a potential marker for progression and treatment response as highlighted in depth in recent reviews. ${ }^{16,17}$

\section{Glutamine as an Emerging Pharmacologic Target}

Due to the demonstrated role of glutamine in gliomas, glutamine metabolism is an emerging pharmacologic target, and several compounds targeting glutamine intake or enzymes involved in glutamine catabolism are currently being evaluated in preclinical and early-stage clinical trials. ${ }^{16}$ Glutaminase, the primary enzyme that catabolizes glutamine in cells, is the target of both telaglenastat or CB-839 and the small-molecule inhibitor compound 968, both of which have been shown to decrease glioma growth, though CB-839 is more specific to glutaminase. ${ }^{11}$ In vitro studies of glutaminase inhibition show depletion of treatment-resistant glioblastoma cells and subsequent reduction of glutamine for downstream use in the TCA cycle. ${ }^{11}$ Because these inhibitors are not as effective against gliomas that exhibit a glycolytic phenotype, ${ }^{10}$ identifying patients with high levels of tumoral glutamine may aid in the prognostication and stratification of patients, particularly for emerging treatments such as glutamine pathway inhibitors. CB-839 is currently being evaluated in a Phase Ib clini$\mathrm{cal}$ trial in combination with chemotherapy and radiation for treatment of IDH-mutant astrocytomas and anaplastic astrocytomas. ${ }^{18}$ A promising glutamine antagonist, 6-diazo-5-oxo-L-norleucin, has also shown efficacy in inhibiting glutaminolysis in humans. ${ }^{16}$ While these treatments hold promise, the standard of care treatment for gliomas is maximal surgical excision followed by radiation therapy with concurrent and adjuvant chemotherapy.

\section{Characterizing and Quantifying Glutamine Metabolism}

Quantifying glutamine in vivo using translational imaging techniques may be useful in identifying glutamine as a biomarker, stratifying patients for novel therapies particularly inhibitors of glutamine metabolism, and accelerating translation of emerging treatments to the clinic. While in vitro experiments are the first step in validating novel treatments targeting glutamine metabolism, they do not fully capture the heterogeneity and complexity of the local tumor environment. ${ }^{19}$ Metabolic phenotypes depend on dynamic interactions between cancer cells and surrounding cells, tissue type, nutrient availability, inflammatory cells, and genetic landscape; in vivo glutamine monitoring is necessary to fully understand its role as a potential prognostic biomarker. Advances in metabolic imaging modalities including MRS, PET, SPECT, and emerging preclinical techniques such as mass spectrometry imaging (MSI) and fluorescence imaging facilitate in vivo measurements of glutamine metabolism in gliomas. Advantages and limitations of imaging techniques capable of measuring glutamine are reviewed below within the context of glioma management.

\section{Glutamine Imaging Techniques}

${ }^{1} \mathrm{H}-\mathrm{MRS}$. MR imaging is a key diagnostic method for gliomas and provides anatomic information on tumor size and location. MRS is a complementary but less used method capable of noninvasively quantifying multiple metabolites simultaneously using the same MR imaging hardware. ${ }^{20}$ MRS does not require an exogeneous contrast agent with the exception of hyperpolarized MRS (see "Hyperpolarized ${ }^{13} \mathrm{C}-\mathrm{MRS}$ ") and relies most commonly on endogenous ${ }^{1} \mathrm{H}$ nuclei. Current MRS research in gliomas is largely focused on detection of 2-HG as a marker for IDH mutations along with decreased NAA and increased choline, lactate, and lipids. ${ }^{20}$ Metabolites related to glutamine metabolism can also be detected using MRS, including glutamine, glutamate, alanine, and glutathione. ${ }^{20}$ One advantage of MRS is the ability to use its ex vivo and in vitro analog, nuclear MR (NMR) spectroscopy, to identify and evaluate promising biomarkers before clinical translation. NMR spectroscopy can detect low metabolite concentrations and overlapping peaks not possible with in vivo MRS and has been used to show that increases in alanine, glutamate, and lactate correlate with lower survival and higher tumor grade in intact glioma tissue. ${ }^{21}$

In vivo, MRS is capable of quantifying localized metabolite concentrations as low as $\sim 1 \mathrm{mM}$ on the minute timescale (Fig $1 A-C$ ). ${ }^{22}$ Tumor concentrations of glutamine measured at $7 \mathrm{~T}$ as high as $5.5 \mathrm{mM}$ have been reported in patients with gliomas. ${ }^{23}$ Glutamine concentrations measured with MRS in normal-appearing contralateral white matter in patients with glioblastoma have been reported to be $\sim 3.4 \mathrm{mM}$, significantly higher than in healthy 


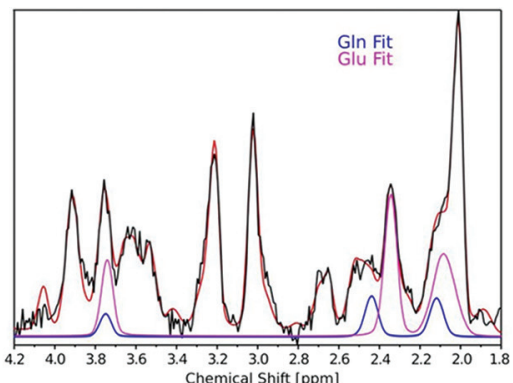

A

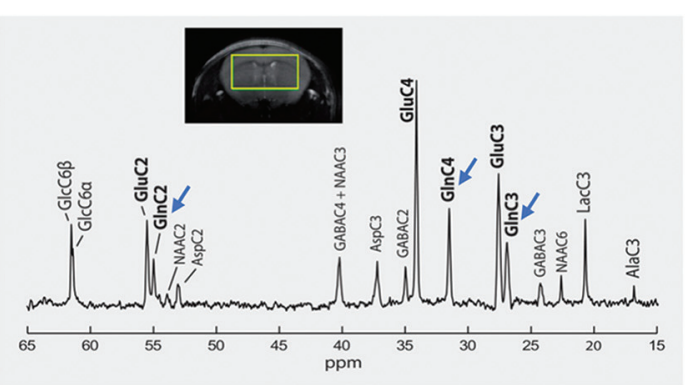

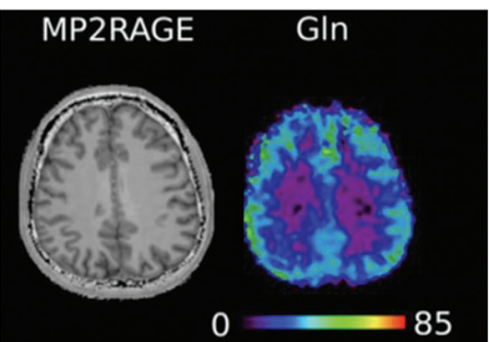

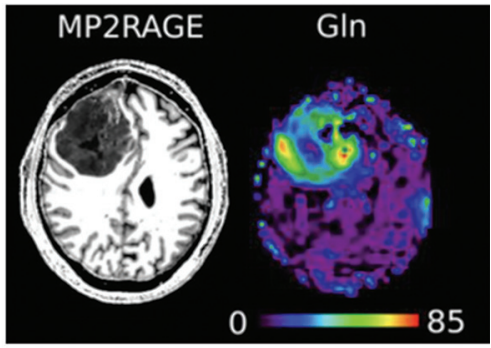

C

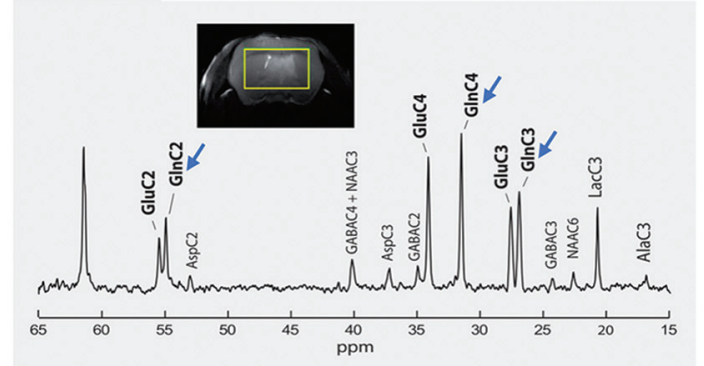

FIG 1. ${ }^{1} \mathrm{H}$ - and ${ }^{13} \mathrm{C}-\mathrm{MR}$ spectra of healthy brain and glioma tissue. A, ${ }^{1} \mathrm{H}-\mathrm{MR}$ spectrum acquired at $7 \mathrm{~T}$ using a $3 \mathrm{D}$ MR spectroscopic imaging sequence in a healthy human volunteer. Glutamine and glutamate peak fits are shown in blue and pink, respectively, demonstrating separation at 7T. B, Structural MP2RAGE image and glutamine metabolite map acquired in the same subject as in A. The Color bar shows metabolite concentrations in arbitrary units. C, Structural MP2RAGE image and a glutamine metabolite map acquired in a male patient with glioblastoma ( 51 years of age). The Color bar shows metabolite concentrations in arbitrary units. Glutamine concentrations are increased in the tumor region, indicated by the dark mass in the MP2RAGE image, relative to the rest of the brain. $A-C$ adapted and reprinted with permission from Hingerl et al. ${ }^{22} \mathrm{D},{ }^{13} \mathrm{C}$-MR spectrum acquired in a healthy mouse brain at $14.1 \mathrm{TT}$ after 3 hours of infusion with $\left[1,6-{ }^{13} \mathrm{C}\right]$-glucose. E, ${ }^{13} \mathrm{C}-\mathrm{MR}$ spectrum acquired from a tumor-bearing mouse brain after 3 hours of infusion of $\left[1,6{ }^{13} \mathrm{C}\right]$-glucose. Decreased glutamate peak intensities (carbon $[\mathrm{C}] 2$, C3, and C4) and glutamine peak intensities ( $\mathrm{C} 2, \mathrm{C} 3$, and $\mathrm{C} 4)$ are observed compared with healthy brain without tumor (blue arrows). D and $E$ adapted and reprinted with permission from Lai et al. ${ }^{30}$ Ala indicates alanine; Asp, aspartate; GABA, $\gamma$-aminobutyric acid; Glc, glucose; Gln, glutamine; Glu, glutamate; Lac, lactate; NAA, N-acetylaspartate.

controls $(2.7 \mathrm{mM})$ or patients with low-grade gliomas $(2.4 \mathrm{mM})$, suggesting the ability of MRS to detect early infiltration in normalappearing regions. ${ }^{24}$ Single-voxel MRS $\left(\sim 8 \mathrm{~cm}^{3}\right)$ and multivoxel MRS are the most common clinical implementations available on many MR imaging scanners, but whole-brain MRS has also been demonstrated in gliomas. ${ }^{25}$ MRS has been used to quantify changes in metabolites as a result of metabolic reprogramming, differentiate proliferative gliomas from brain metastasis, and predict tumor grade. ${ }^{25,26}$ Longitudinal ${ }^{1} \mathrm{H}$-MRS studies in patients with glioblastomas showed that pretreatment increases in glutamine plus glutamate were correlated with tumor proliferation and poor prognosis. $^{27}$

While MRS has the benefits of repeatability, lack of ionizing radiation, and availability at many clinical sites, separating overlapping glutamine and glutamate resonances in ${ }^{1} \mathrm{H}-\mathrm{MR}$ spectra acquired at typical clinical field strengths (1.5T or $3 \mathrm{~T})$ can be challenging. ${ }^{20}$ The commonly used point-resolved spectroscopy (PRESS) sequence with a TE of $20 \mathrm{~ms}$ at $3 \mathrm{~T}$ has been shown to detect both glutamine and glutamate with quantification comparable with that of spectra acquired on 7T MR imaging scanners; ${ }^{28}$ however, higher-field-strength scanners facilitate improved signal-to-noise ratios and better peak separation. ${ }^{29}$ In vivo studies using high-field MR imaging scanners have demonstrated significantly higher glutamine levels in glioma-bearing mice at the onset of neurologic symptoms compared with presymptomatic mice. ${ }^{30}$ With recent FDA approval of both Siemens (Magnetom Terra) and GE (Signa) 7T MR scanners, MRS has the potential to become a viable method for routine glutamine imaging.

Further advances in ${ }^{1} \mathrm{H}$-MRS methods have improved the detection of glutamine and separation from glutamate peaks. Chemical exchange saturation transfer, which measures chemical exchange between bulk water and metabolites, has been shown to facilitate quantification of glutamate in response to CB-839 treatment in breast cancer, ${ }^{31}$ and glutamine chemical exchange saturation transfer has been used for $\mathrm{pH}$ monitoring in animal models of brain tumors. ${ }^{32}$ Spectral editing techniques can separate overlapping peak resonances in 1D spectra and have been used to detect $\gamma$-aminobutyric acid, 2-HG, and glutathione. ${ }^{33-35}$ Most spectral editing techniques use $J$-difference editing to isolate the signal of interest. For example, a $J$-modulated spectroscopy method was shown to separate glutamine from glutamate in spectra acquired at $3 \mathrm{~T}$ in healthy adult brains. ${ }^{35}$ Emerging single-step spectral editing methods have been demonstrated for simultaneous quantification of glutamine, glutamate, and glutathione at 7T in healthy adults. ${ }^{34}$ Because increased glutamine and glutamate in gliomas correlate with increased proliferation and glutathione is implicated in treatment resistance, further evaluation in patients with gliomas undergoing treatment is warranted. ${ }^{34,36}$ While 2D techniques, including localized correlational spectroscopy and J-PRESS, have also enabled separation of glutamine from glutamate, ${ }^{37}$ long scan times have relegated many $2 \mathrm{D}$ sequences to research applications. 

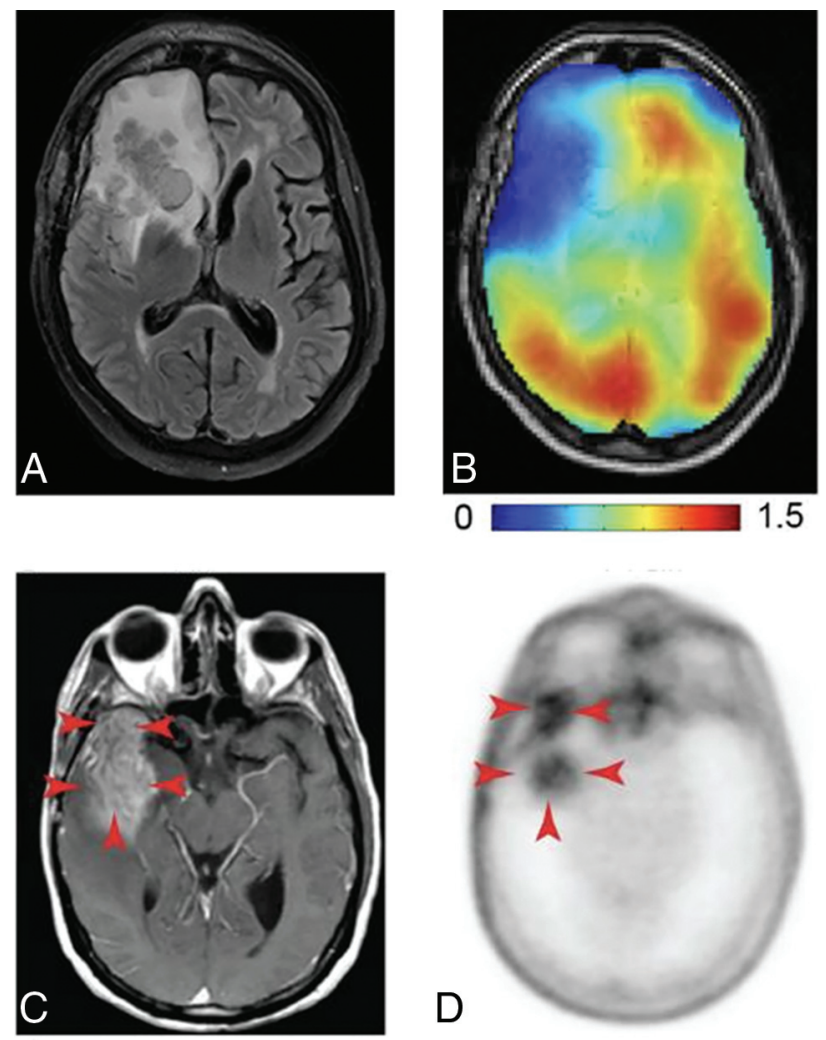

0

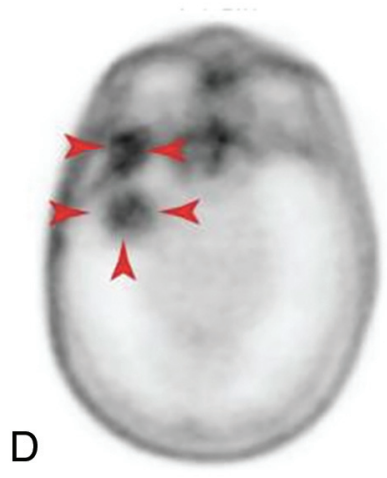

FIG 2. DMI and PET images of glutamine metabolism in gliomas. A, T2-weighted FLAIR MR image acquired in a patient with a glioblastoma with a tumor in the right frontal lobe. $B$, DMI maps of the same section position as in $A$ showing ${ }^{2} \mathrm{H}$-labeled glutamine + glutamate, with lower concentrations in the tumor region compared with normal-appearing brain tissue. Color bar is in millimolar units. $A$ and $B$ reprinted with permission from DeFeyter et $\mathrm{al}^{41}$ and used under the CC BY-NC license 4.0. C, T7-weighted MR image with contrast enhancement acquired in a patient with glioblastoma. The tumor region is indicated with red arrows. D, 4- $\left[{ }^{18} \mathrm{~F}\right]$-FGln PET image acquired in the same patient as in $C$, with high uptake in the tumor region (red arrows) and minimal background uptake. $C$ and $D$ reprinted from Venneti et al, ${ }^{13}$ with permission from AAAS.

Hyperpolarized ${ }^{13} \mathrm{C}$-MRS. ${ }^{13} \mathrm{C}$-MRS has been used to study the brain; however, due to low sensitivity from limited natural abundance and a low gyromagnetic ratio, hyperpolarized ${ }^{13} \mathrm{C}$ is required in vivo. Hyperpolarization increases ${ }^{13} \mathrm{C}$-signal temporarily via dynamic nuclear polarization. ${ }^{38}$ After infusion with a hyperpolarized ${ }^{13} \mathrm{C}$-labeled substrate, the flux of the labeled substrate to its metabolic products can be measured (Fig $1 D,-E){ }^{30}$ One of the most commonly studied ${ }^{13} \mathrm{C}$ metabolites is $\left[1-{ }^{13} \mathrm{C}\right]$-pyruvate, which is catabolized into lactate, alanine, and bicarbonate in cells and provides a useful tool to monitor the by-products of glycolysis. ${ }^{38}\left[1-{ }^{13} \mathrm{C}\right]$-pyruvate has been used in patients with gliomas to measure the conversion of pyruvate to lactate, with high rates of lactate production observed in recurrent tumors. ${ }^{39}$ Hyperpolarized ${ }^{13} \mathrm{C}$ tracers have been developed for glucose, acetate, $\alpha$-ketoglutarate, and glutamine, all of which have demonstrated blood-brain barrier permeability necessary for hyperpolarized MRS experiments. ${ }^{38}{ }^{13} \mathrm{C}$-MRS has been used to assess changes in glutamine metabolism in response to treatments. Subramani et $\mathrm{al}^{14}$ showed that glioma-bearing mice injected with $\left[3-{ }^{13} \mathrm{C}\right]$-glutamine convert glutamine to glutamate at higher rates after treatment with temozolomide. This increase in glutamate was also observed using ${ }^{1} \mathrm{H}-\mathrm{MRS}$ on the same mice. ${ }^{14}$ Similarly, Molloy et $\mathrm{al}^{40}$ used $\left[3-{ }^{13} \mathrm{C}\right]$-glutamine and demonstrated an increase in conversion of glutamine to glutamate in glioma cells in response to a downstream inhibitor of glutamine metabolism. These studies highlight the potential of hyperpolarized ${ }^{13} \mathrm{C}$-MRS studies to monitor glutamine metabolites both for validating ${ }^{1} \mathrm{H}-\mathrm{MRS}$ findings and evaluating the response to treatment. While an on-site hyperpolarizer is required and short $\mathrm{T} 1$ relaxation times require rapid imaging for most ${ }^{13} \mathrm{C}$ tracers, ${ }^{13} \mathrm{C}$-MRS facilitates direct measurement of glutamine metabolism without the use of an ionizing tracer.

Deuterium Metabolic Imaging. In vivo deuterium metabolic imaging (DMI) is an emerging MRS technique that measures ${ }^{2} \mathrm{H}$ labeled substrates. ${ }^{2} \mathrm{H}$-MRS has been primarily demonstrated to detect labeled glucose and its metabolic products including glutamine, glutamate, and lactate (Fig $2 A,-B) .{ }^{41}$ In rats and humans, DMI has revealed metabolic differences between gliomas and healthy tissue, with glioma tissue exhibiting lower levels of glutamine plus glutamate and higher levels of lactate and glucose due to the Warburg effect. ${ }^{41}$ While DMI has been used in vivo to image and quantify glucose metabolism and flux through the TCA cycle, separation of glutamine and glutamate, even at high magnetic field strengths, is challenging. Moreover, the gyromagnetic ratio of ${ }^{2} \mathrm{H}$ is $\sim 7$ times lower than ${ }^{1} \mathrm{H}$ and requires ingestion or injection of a ${ }^{2} \mathrm{H}$-labeled substrate. DMI technology is improving quickly and provides a noninvasive method of quantifying active metabolism in vivo. ${ }^{42}$

MRS Limitations and Considerations. MRS, while not standard clinical practice, is frequently used in patients with gliomas. Currently, it is most often ordered by clinicians to characterize increasing enhancement after previous radiation therapy and to distinguish radiation-induced necrosis from recurrent tumor. Limitations of MRS include nonstandardized analysis, challenges with reproducibility, the need for input by the radiologist for voxel placement, low signal-to-noise ratios, and long acquisition times. Optimization of in vivo glutamine quantification with MRS, combined with improved postprocessing methods, is an immediate goal. ${ }^{43,44}$ Fast, whole-brain MRS to generate spatial glutamine maps is likely necessary before incorporation of MRS into routine clinical workflow. Most important, MRS, while FDA-approved, is still considered investigational by most insurance companies, and lack of reimbursement has limited widespread clinical implementation. As more data emerge regarding the value of quantifying glutamine for gliomas, MRS will be increasingly recognized and recommended by clinicians and radiologists, possibly working to counter this issue. For a comprehensive review of clinical MRS, we direct readers to de Graaf et $\mathrm{al}^{42}$ and Wilson et al. ${ }^{43}$

Glutamine PET. FDG-PET has been used clinically for tumor grading, determining tumor boundaries, and guiding biopsies. ${ }^{45}$ PET has high sensitivity and spatial and temporal resolution $(3-5 \mathrm{~mm}$ on the second timescale); however, specificity is limited by uptake of FDG in healthy brain as well as tumors. ${ }^{45}$ A recently developed glutamine fluoro-analog, $4-\left[{ }^{18} \mathrm{~F}\right]-(2 \mathrm{~S}, 4 \mathrm{R})$-fluoroglutamine $\left(\left[{ }^{18} \mathrm{~F}\right]\right.$ FGln) has enabled noninvasive imaging of glutamine in gliomas 
(Fig 2C, $-D) .{ }^{13}$ Mice and humans with gliomas show high $\left[{ }^{18} \mathrm{~F}\right]-$ FGln uptake in tumors, and $\left[{ }^{18} \mathrm{~F}\right]$-FGln PET may distinguish actively growing gliomas from stable tumors. ${ }^{13}$ In a study of mice with gliomas, tumor-to-background ratios of 3.6 were observed, indicating increased $\left[{ }^{18} \mathrm{~F}\right]$-FGln in tumor compared with healthy tissue. $^{46}$ A study of 14 patients with cancer metastasis to the brain showed significantly higher $\left[{ }^{18} \mathrm{~F}\right]$-FGln PET activity in the tumor relative to healthy brain tissue compared with FDG-PET, indicating the potential utility of glutamine PET. ${ }^{47}$ In a Phase I clinical trial, $\left[{ }^{18} \mathrm{~F}\right]$-FGln PET was able to identify tumors with genetic precursors to glutamine addiction, including $I D H$ and tumor protein 53 mutations in several cancers including gliomas. ${ }^{48}$

Glutamine PET tracers may be advantageous for identifying gliomas exhibiting glutamine addiction and stratifying patients for treatment. In mice, gliomas treated with chemotherapy exhibited significantly decreased $\left[{ }^{18} \mathrm{~F}\right]$-FGln uptake compared with pretreatment tumors. ${ }^{13}$ Similar results were also observed in mice with breast, colorectal, and lung cancer treated with the V-9302, an inhibitor of glutamine transporter ASCT2. ${ }^{49}$ However, pharmacokinetic studies indicate that $\left[{ }^{18} \mathrm{~F}\right]-\mathrm{FGln}$ is defluorinated and metabolized quickly. ${ }^{46}\left[{ }^{18} \mathrm{~F}\right]$-FGln derivatives have been developed to improve the stability of the tracers in vivo to address this limitation. $\left[{ }^{18} \mathrm{~F}\right]$-fluoroboronoglutamine has improved stability in mice, though the efficacy in glioma models is yet to be determined. ${ }^{50}$

In addition to $\left[{ }^{18} \mathrm{~F}\right]-\mathrm{FGln}$, two dipeptide glutamine PET tracers, $\left[{ }^{18} \mathrm{~F}\right]$-Gly- $(2 S, 4 R) 4$-fluoroglutamine and $\left[{ }^{18} \mathrm{~F}\right]$-Ala- $(2 S, 4 R) 4$ fluoroglutamine, have been developed with improved in vivo stability. After injection of the tracers in mice with gliosarcomas, tracers were localized in the tumors and $\left[{ }^{18} \mathrm{~F}\right] \mathrm{Ala}-(2 S, 4 R) 4$-fluoroglutamine displayed a similar distribution and tumor-to-background ratio compared with $\left[{ }^{18} \mathrm{~F}\right]$-FGln. $\left[{ }^{18} \mathrm{~F}\right]$-Ala- $(2 S, 4 R) 4-$ fluoroglutamine may be comparable with the $\left[{ }^{18} \mathrm{~F}\right]$-FGln PET tracer; however, further validation in clinical trials is necessary. ${ }^{51}$ In addition to $\left[{ }^{18} \mathrm{~F}\right]-\mathrm{FGln}$, the ${ }^{11} \mathrm{C}$-glutamine PET tracer $5_{-}{ }^{11} \mathrm{C}$ (2S)-glutamine $\left({ }^{11} \mathrm{C}\right.$-Gln $)$ has also been developed. In the first inhuman study, Cohen et $\mathrm{al}^{52}$ evaluated the utility of ${ }^{11} \mathrm{C}-\mathrm{Gln}$ in tracking treatment response to $\mathrm{CB}-839$ as part of a clinical trial evaluating glutamine inhibitors for treatment of colorectal cancer. Preliminary results revealed the utility of ${ }^{11} \mathrm{C}$-Gln in identifying heterogeneous glutamine uptake by lesions; however, the intrinsically shorter half-life of ${ }^{11} \mathrm{C}$-Gln compared with $\left[{ }^{18} \mathrm{~F}\right]$-FGln may limit its utility. Further comparisons of the in vivo kinetics of $\left[{ }^{18} \mathrm{~F}\right]-\mathrm{FGln}$ and ${ }^{11} \mathrm{C}-\mathrm{G} \ln$ tracers in patients with gliomas will be important for determining the utility of ${ }^{11} \mathrm{C}-\mathrm{Gln}$.

PET Limitations and Considerations. While PET may be useful in different stages of glioma care because it provides direct and quantitative information about glutamine metabolism, limitations include the short half-life of labeling isotopes $\left(\mathrm{eg},{ }^{11} \mathrm{C}\right)$ requiring an on-site cyclotron, exposure to ionizing radiation, and analysis possibly requiring measurements of labeled glucose or glutamine levels in arterial blood. Many of these limitations can be overcome by development of glutamine analogues labeled with longer-lived isotopes (eg, $\left[{ }^{18} \mathrm{~F}\right]$ ) and by using internal reference regions or image-derived input functions to negate the need for arterial blood in the analysis. With the advent of large-FOV PET systems, the increased quantitative sensitivity offered by a

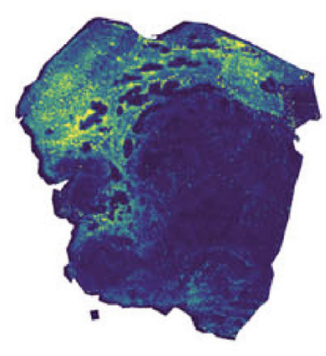

Intensity (arb. unit) $0 \% \quad 100 \%$

FIG 3. MSI to image glutamine metabolism. Matrix-assisted laser desorption/ionization TOF MSI of glutamine distribution in an ex vivo human glioma sample (left), with an annotated brightfield image of the same sample showing the tumor and peritumoral regions (right). The MSI map shows increased intensity of glutamine in the tumor regions compared with both peritumoral and nontumoral regions and is a promising emerging method for imaging glutamine. Arb. unit indicates arbitrary unit. Reprinted with permission from Kampa et $\mathrm{al}^{56}$ and used under the CC BY license 4.0.

full kinetic analysis will become more accessible in the clinical environment. ${ }^{53}$ While $\left[{ }^{18} \mathrm{~F}\right]$-FGln and ${ }^{11} \mathrm{C}$-Gln tracers are not yet approved by the FDA, FDA approval of amino acid-based PET tracers support the potential and feasibility of glutamine PET, ${ }^{54}$ and preliminary work has shown that these agents can discern low- from high-grade gliomas. ${ }^{55}$ Ongoing clinical trials will continue to elucidate the safety and efficacy of glutamine PET for gliomas. Further development and validation studies will be increasingly important as glutamine continues to emerge as a marker of prognosis and treatment response.

\section{Emerging Methods}

Emerging methods for glutamine detection, including MSI, fluorescence imaging, and glutamine SPECT, have shown utility in characterizing glutamine metabolism in animal models and may complement clinically integrated methods such as MR imaging and PET. MSI quantifies the spatial distribution of metabolites including glutamine by conducting mass spectrometry experiments of thin tissue slices (Fig 3). ${ }^{56}$ MSI of human glioblastoma samples shows significant increases in glutamine, glutamate, and lactate in the tumor region compared with peritumoral and nontumoral regions. ${ }^{56}$ Advanced MSI methods including ultra-highresolution MSI based on Fourier-transform mass spectrometry also reveal heterogeneous distributions of metabolites related to both the TCA cycle and glutamine metabolism in mouse glioblastoma tissue. ${ }^{57}$ Another application of MSI is identifying differences in the energetics of glioma molecular subtypes in patientderived xenografts, which reveal that $I D H$-mutated gliomas have abnormal mitochondrial metabolism compared with $I D H$ wildtype gliomas yet do not have significantly different glutamine or glutamate levels. ${ }^{58}$ MSI has high molecular specificity and spatial resolution (submillimeter); however, it is currently performed only on ex vivo samples, and acquisition times are long (minutes to hours). While quantifying glutamine in excised tissue is a 


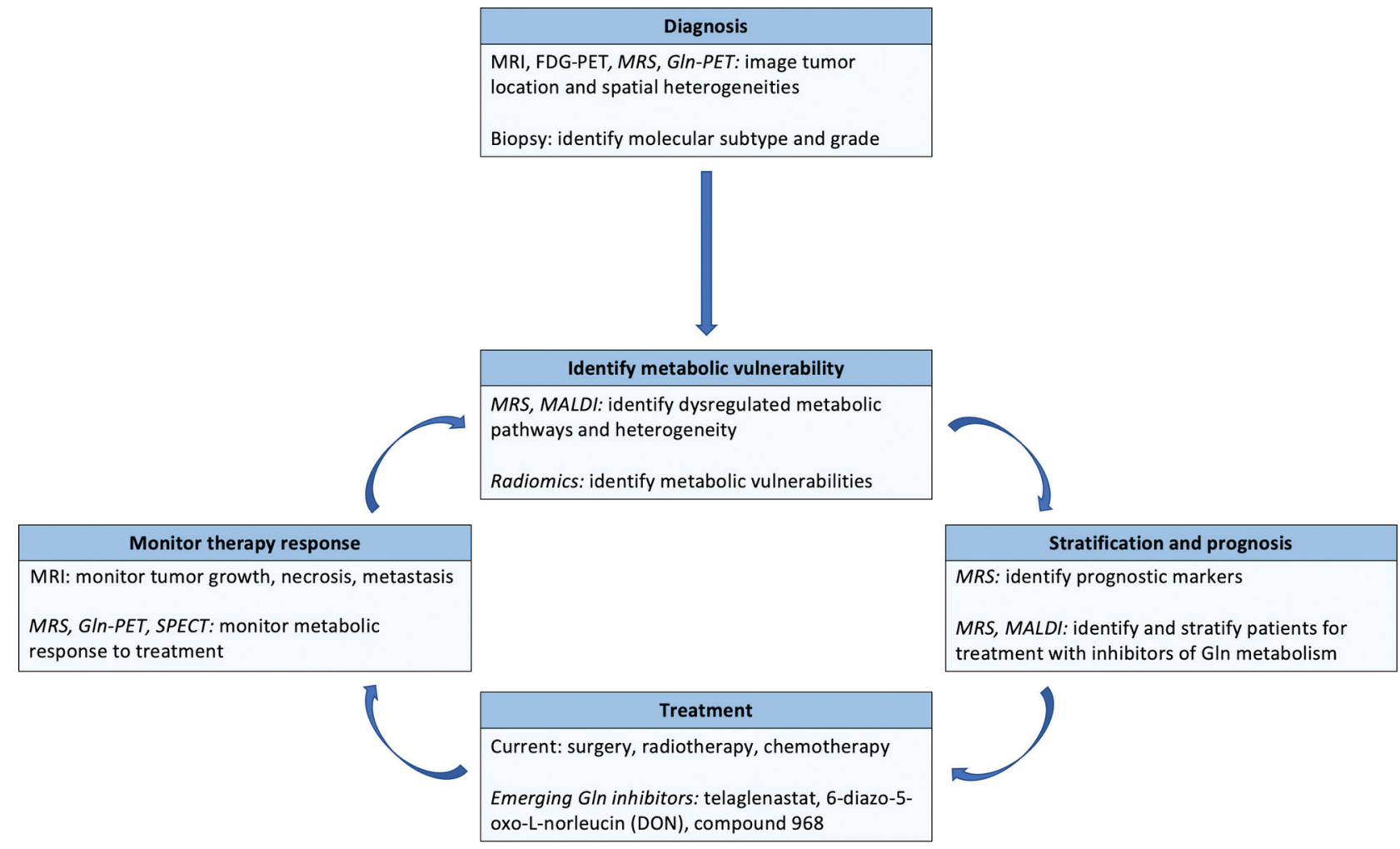

FIG 4. Framework for using glutamine imaging techniques in glioma management. Imaging methods to quantify and measure local glutamine changes may be useful for diagnosis, identification of metabolic vulnerabilities, patient stratification, and improved treatment monitoring. Methods currently not integrated into clinical workflows are indicated with italics. MALDI indicates matrix-assisted laser desorption/ionization.

limitation of MSI compared with MR imaging and PET because excised tissue may undergo degradation before metabolite quantification, ${ }^{59}$ its high specificity may be used to validate results from MR imaging and PET studies. Furthermore, analysis of excised tissue may also aid pathologists and cancer biologists in characterizing the considerable intratumoral heterogeneity of glioblastoma. ${ }^{60}$

Fluorescence imaging of glutamine and glutamine transporters using optical microscopy and spectroscopy is an emerging method with high spatial resolution (submicron), currently limited to in vitro studies. ${ }^{61,62}$ Quantification of glutamine uptake and energy flux has been demonstrated in brain cancer cells in response to epidermal growth factor receptor treatment. ${ }^{63}$ Although this technology currently analyzes a small number of cells, proof-of-concept studies to characterize the glutamine response to new treatments are an important first step to motivate larger in vivo studies. Last, new glutamine SPECT tracers have been developed and show promise as clinical tools. Ghoreishi et $\mathrm{al}^{64}$ conjugated glutamine to technetium Tc99m, a commonly used isotope for SPECT. Mice injected with the nanoconjugate tracer showed significantly higher radioactivity in lung tumors compared with other organs and high stability in human serum. While SPECT is clinically integrated and both fluorescence imaging of glutamine transport and SPECT glutamine tracers are promising, evaluation of these emerging methods in humans is unexplored.

\section{Discussion and Outlook}

Targeting glutamine metabolism for improved outcomes in patients with gliomas holds clinical promise, particularly as emerging pharmacologic treatments targeting glutamine catabolism and transport have shown efficacy in vivo (Fig 4). ${ }^{8}$ In vivo glutamine quantification is clinically achievable due to advances in noninvasive image acquisition and analysis. Previous studies have shown that glutamine imaging with ${ }^{1} \mathrm{H}-\mathrm{MRS},{ }^{13} \mathrm{C}-\mathrm{MRS}$, and PET has potential prognostic value because concentrations of glutamine and its related metabolites are correlated with decreased survival. ${ }^{12,13,30}$ Emerging techniques including MSI and fluorescence imaging may validate MR imaging and PET findings at the single-cell level. ${ }^{57,62}$

In the future, multiple strategies may facilitate evaluation of glutamine as a prognostic marker. The first and most straightforward strategy is the continued standardization and development of MRS and PET for glutamine quantification because both modalities are frequently used in clinical cancer imaging. The second is the use of multiparametric approaches, including radiomics, to use existing clinical data to improve prediction and stratification. ${ }^{65}$ Incorporation of metabolic information, including glutamine into radiomics analyses, has been limited; however, radiomics may facilitate the use of the large amount of imaging data often untouched in radiologic practice. The final strategy is advancement of emerging molecular-scale methods, including those outlined here. Given the high cost and limited availability of clinical imaging systems particularly in rural areas, development of low-cost methods is an important goal for the future.

Although glutamine imaging is still largely in the research and development stage, monitoring changes in glutamine metabolism may provide insight into tumor heterogeneity and response to new therapies, enabling stratification for personalized treatment 
plans. The development of new treatment strategies, particularly pharmacologic compounds targeting the glutamine pathway, will likely benefit from longitudinal metabolic imaging that includes quantification of local glutamine concentrations. While outstanding challenges in glutamine imaging must be addressed, including standardization and, in some cases, FDA approval, there is clear motivation for the continued investigation and evaluation of glutamine as a promising imaging biomarker of glioma progression, treatment selection and stratification, and prognosis.

Disclosures: Selin Ekici-UNRELATED: Travel/Accommodations/Meeting Expenses Unrelated to Activities Listed: International Society for Magnetic Resonance in Medicine annual conferences, Comments: awarded a waived registration and trainee (educational) stipend for the 2019 and 2020 annual meetings. Jonathon A. Nye-UNRELATED: Employment: Emory University School of Medicine.

\section{REFERENCES}

1. Reni M, Mazza E, Zanon S, et al. Central nervous system gliomas. Crit Rev Oncol Hematol 2017;113:213-34 CrossRef Medline

2. Pavlova NN, Thompson CB. The emerging hallmarks of cancer metabolism. Cell Metab 2016;23:27-47 CrossRef Medline

3. Hensley CT, Wasti AT, DeBerardinis RJ. Glutamine and cancer: cell biology, physiology, and clinical opportunities. J Clin Invest 2013;123:3678-84 CrossRef Medline

4. Márquez J, Alonso FJ, Matés JM, et al. Glutamine addiction in gliomas. Neurochem Res 2017;42:1735-46 CrossRef Medline

5. Vander Heiden MG, DeBerardinis RJ. Understanding the intersections between metabolism and cancer biology. Cell 2017;168:65769 CrossRef Medline

6. $\mathrm{Hu} \mathrm{S}$, Balakrishnan $\mathrm{A}$, Bok RA, et al. 13C-pyruvate imaging reveals alterations in glycolysis that precede c-Myc-induced tumor formation and regression. Cell Metab 2011;14:131-42 CrossRef Medline

7. Nicklin P, Bergman P, Zhang B, et al. Bidirectional transport of amino acids regulates mTOR and autophagy. Cell 2009;136:521-34 CrossRef Medline

8. Li T, Copeland C, Le A. Glutamine metabolism in cancer. In: Le A, ed. The Heterogeneity of Cancer Metabolism. Springer-Verlag International Publishing; 2021:17-38

9. Cluntun AA, Lukey MJ, Cerione RA, et al. Glutamine metabolism in cancer: understanding the heterogeneity. Trends Cancer 2017;3:16980 CrossRef Medline

10. Oizel K, Chauvin C, Oliver L, et al. Efficient mitochondrial glutamine targeting prevails over glioblastoma metabolic plasticity. Clin Cancer Res 2017;23:6292-6304 CrossRef Medline

11. Koch K, Hartmann R, Tsiampali J, et al. A comparative pharmacometabolomic study of glutaminase inhibitors in glioma stem-like cells confirms biological effectiveness but reveals differences in target-specificity. Cell Death Discov 2020;6:20 CrossRef Medline

12. Oizel K, Yang C, Renoult $\mathrm{O}$, et al. Glutamine uptake and utilization of human mesenchymal glioblastoma in orthotopic mouse model. Cancer Metab 2020;8:9 CrossRef Medline

13. Venneti S, Dunphy MP, Zhang H, et al. Glutamine-based PET imaging facilitates enhanced metabolic evaluation of gliomas in vivo. Sci Transl Med 2015;7:274ra17 CrossRef Medline

14. Subramani E, Radoul M, Najac C, et al. Glutamate is a noninvasive metabolic biomarker of IDH1-mutant glioma response to temozolomide treatment. Cancer Res 2020;80:5098-5108 CrossRef Medline

15. Byun JK, Park M, Lee S, et al. Inhibition of glutamine utilization synergizes with immune checkpoint inhibitor to promote antitumor immunity. Mol Cell 2020;80:592-606.e8 CrossRef Medline

16. Choi YK, Park KG. Targeting glutamine metabolism for cancer treatment. Biomol Ther (Seoul) 2018;26:19-28 CrossRef Medline

17. Altman BJ, Stine ZE, Dang CV. From Krebs to clinic: glutamine metabolism to cancer therapy. Nat Rev Cancer 2016;16:619-34 CrossRef Medline
18. Kizilbash SH, McBrayer S, Port J, et al. A phase Ib trial of CB-839 (telaglenastat) in combination with radiation therapy and temozolomide in patients with IDH-mutated diffuse astrocytoma and anaplastic astrocytoma (NCT03528642). J Clin Oncol 2019;37:TPS2075 CrossRef

19. Lenting K, Verhaak R, Ter Laan M, et al. Glioma: experimental models and reality. Acta Neuropathol 2017;133:263-82 CrossRef Medline

20. Öz G, Alger JR, Barker PB, et al; MRS Consensus Group. Clinical proton MRS in central nervous system disorders. Radiology 2014;270:658-79 CrossRef Medline

21. Ekici S, Risk BB, Neill SG, et al. Characterization of dysregulated glutamine metabolism in human glioma tissue with 1H NMR. Sci Rep 2020;10:20435 CrossRef Medline

22. Hingerl L, Strasser B, Moser P, et al. Clinical high-resolution 3DMR spectroscopic imaging of the human brain at $7 \mathrm{~T}$. Invest Radiol 2020;55:239-48 CrossRef Medline

23. Ganji SK, An Z, Tiwari V, et al. In vivo detection of 2-hydroxyglutarate in brain tumors by optimized point-resolved spectroscopy (PRESS) at 7T. Magn Reson Med 2017;77:936-44 CrossRef Medline

24. Kallenberg K, Bock HC, Helms G, et al. Untreated glioblastoma multiforme: increased myo-inositol and glutamine levels in the contralateral cerebral hemisphere at proton MRS. Radiology 2009;253:805-12 CrossRef Medline

25. Cordova JS, Shu HK, Liang Z, et al. Whole-brain spectroscopic MRI biomarkers identify infiltrating margins in glioblastoma patients. Neuro Oncol 2016;18:1180-89 CrossRef Medline

26. Ohba S, Murayama K, Abe M, et al. Magnetic resonance imaging and proton magnetic resonance spectroscopy for differentiating between enhanced gliomas and malignant lymphomas. World Neurosurg 2019;127:e779-87 CrossRef Medline

27. Heintz A, Chombar J, Boussida S, et al. P14.92 study of glutaminergic and glutamatergic metabolism in 1H-MRS monovoxel in the most aggressive part of 62 glioblastoma before and after 18 months treatment. Neuro Oncol 2019;21(Suppl 3):iii89 CrossRef

28. Bell T, Goerzen D, Near J, et al. Examination of methods to optimize glutamate-glutamine separation at 3T. In: Proceedings of the International Society for Magnetic Resonance in Medicine, Virtual. August 8-14, 2020

29. Pradhan S, Bonekamp S, Gillen JS, et al. Comparison of single voxel brain MRS at $3 \mathrm{~T}$ and $7 \mathrm{~T}$ using 32-channel head coils. Magn Reson Imaging 2015;33:1013-18 CrossRef Medline

30. Lai M, Vassallo I, Lanz B, et al. In vivo characterization of brain metabolism by $1 \mathrm{H}$ MRS, 13C MRS and 18FDG PET reveals significant glucose oxidation of invasively growing glioma cells. Int $J$ Cancer 2018;143:127-38 CrossRef Medline

31. Zhou R, Bagga P, Nath K, et al. Glutamate-weighted chemical exchange saturation transfer magnetic resonance imaging detects glutaminase inhibition in a mouse model of triple-negative breast cancer. Cancer Res 2018;78:5521-26 CrossRef Medline

32. Harris RJ, Cloughesy TF, Liau LM, et al. pH-weighted molecular imaging of gliomas using amine chemical exchange saturation transfer MRI. Neuro Oncol 2015;17:1514-24 CrossRef Medline

33. An L, Araneta MF, Johnson C, et al. Simultaneous measurement of glutamate, glutamine, GABA, and glutathione by spectral editing without subtraction. Magn Reson Med 2018;80:1776-86 CrossRef Medline

34. An L, Araneta MF, Victorino M, et al. Signal enhancement of glutamine and glutathione by single-step spectral editing. J Magn Reson 2020;316:106756 CrossRef Medline

35. Zhang Y, Shen J. Simultaneous quantification of glutamate and glutamine by J-modulated spectroscopy at 3 Tesla. Magn Reson Med 2016;76:725-32 CrossRef Medline

36. Olivier C, Oliver L, Lalier L, et al. Drug resistance in glioblastoma: the two faces of oxidative stress. Front Mol Biosci 2020;7:620677 Medline 
37. Arm J, Al-Iedani O, Quadrelli S, et al. Reliability of neurometabolite detection with two-dimensional localized correlation spectroscopy at 3T. J Magn Reson Imaging 2018;48:1559-69 CrossRef Medline

38. Wang ZJ, Ohliger MA, Larson PE, et al. Hyperpolarized 13C MRI: state of the art and future directions. Radiology 2019;291:273-84 CrossRef Medline

39. Miloushev VZ, Granlund KL, Boltyanskiy R, et al. Metabolic imaging of the human brain with hyperpolarized $13 \mathrm{C}$ pyruvate demonstrates 13C lactate production in brain tumor patients. Cancer Res 2018;78:3755-60 CrossRef Medline

40. Molloy AR, Najac C, Viswanath P, et al. MR-detectable metabolic biomarkers of response to mutant IDH inhibition in low-grade glioma. Theranostics 2020;10:8757-70 CrossRef Medline

41. De Feyter HM, Behar KL, Corbin ZA, et al. Deuterium metabolic imaging (DMI) for MRI-based 3D mapping of metabolism in vivo. Sci Adv 2018;4:eaat7314 CrossRef Medline

42. de Graaf RA, Hendriks AD, Klomp DW, et al. On the magnetic field dependence of deuterium metabolic imaging. NMR Biomed 2020;33: e4235 CrossRef Medline

43. Wilson M, Andronesi O, Barker PB, et al. Methodological consensus on clinical proton MRS of the brain: review and recommendations. Magn Reson Med 2019;82:527-50 CrossRef Medline

44. Sung D, Risk BB, Owusu-Ansah M, et al. Optimized truncation to integrate multi-channel MRS data using rank-R singular value decomposition. NMR Biomed 2020;33:e4297 CrossRef Medline

45. Albert NL, Weller M, Suchorska B, et al. Response Assessment in Neuro-Oncology Working Group and European Association for Neuro-Oncology recommendations for the clinical use of PET imaging in gliomas. Neuro Oncol 2016;18:1199-208 CrossRef Medline

46. Miner MW, Liljenbäck H, Virta J, et al. (2S, 4R)-4-[(18)F] fluoroglutamine for on vivo PET imaging of glioma xenografts in mice: an evaluation of multiple pharmacokinetic models. Mol Imaging Biol 2020;22:969-78 CrossRef Medline

47. $\mathrm{Xu} \mathrm{X}, \mathrm{Zhu} \mathrm{H}$, Liu $\mathrm{F}$, et al. Imaging brain metastasis patients with 18F-(2S,4R)-4-fluoroglutamine. Clin Nucl Med 2018;43:e392-99 CrossRef Medline

48. Dunphy MPS, Harding JJ, Venneti S, et al. In vivo PET assay of tumor glutamine flux and metabolism: in-human trial of (18)F-(2S,4R)-4fluoroglutamine. Radiology 2018;287:667-75 CrossRef Medline

49. Schulte ML, Fu A, Zhao P, et al. Pharmacological blockade of ASCT2-dependent glutamine transport leads to antitumor efficacy in preclinical models. Nat Med 2018;24:194-202 CrossRef Medline

50. Li C, Liu H, Duan D, et al. Preclinical study of an 18F-labeled glutamine derivative for cancer imaging. Nucl Med Biol 2018;64-65:3440 CrossRef Medline

51. Zha Z, Ploessl K, Lieberman BP, et al. Alanine and glycine conjugates of $(2 S, 4 R)-4-[18 F]$ fluoroglutamine for tumor imaging. $\mathrm{Nucl}$ Med Biol 2018;60:19-28 CrossRef Medline
52. Cohen AS, Grudzinski J, Smith GT, et al. First-in-human PET imaging and estimated radiation dosimetry of $\mathrm{L}-\left[5^{-11} \mathrm{C}\right]$-glutamine in patients with metastatic colorectal cancer. J Nucl Med 2021 April 30. [Epub ahead of print] CrossRef Medline

53. Dimitrakopoulou-Strauss A, Pan L, Sachpekidis C. Kinetic modeling and parametric imaging with dynamic PET for oncological applications: general considerations, current clinical applications, and future perspectives. Eur J Nucl Med Mol Imaging 2021;48:21-39 CrossRef Medline

54. Galldiks N, Langen K-J. Amino acid PET: an imaging option to identify treatment response, posttherapeutic effects, and tumor recurrence? Front Neurol 2016;7:120 CrossRef Medline

55. Albano D, Tomasini D, Bonù M, et al. ${ }^{18}$ F-fluciclovine $\left({ }^{18}\right.$ F-FACBC $)$ PET/CT or PET/MRI in gliomas/glioblastomas. Ann $\mathrm{Nucl} \mathrm{Med}$ 2020;34:81-86 CrossRef Medline

56. Kampa JM, Kellner U, Marsching C, et al. Glioblastoma multiforme: metabolic differences to peritumoral tissue and IDH-mutated gliomas revealed by mass spectrometry imaging. Neuropathology 2020;40:546-58 CrossRef Medline

57. Dilillo M, Ait-Belkacem R, Esteve C, et al. Ultra-high mass resolution MALDI imaging mass spectrometry of proteins and metabolites in a mouse model of glioblastoma. Sci Rep 2017;7:603 CrossRef Medline

58. Fack F, Tardito S, Hochart G, et al. Altered metabolic landscape in IDH-mutant gliomas affects phospholipid, energy, and oxidative stress pathways. EMBO Mol Med 2017;9:1681-95 CrossRef Medline

59. Esteve C, Tolner EA, Shyti R, et al. Mass spectrometry imaging of amino neurotransmitters: a comparison of derivatization methods and application in mouse brain tissue. Metabolomics 2016;12:30 CrossRef Medline

60. Bernstock JD, Mooney JH, Ilyas A, et al. Molecular and cellular intratumoral heterogeneity in primary glioblastoma: clinical and translational implications. J Neurosurg 2019 Aug 23 [Epub ahead of print] CrossRef Medline

61. Besnard J, Okumoto S. Glutamine flux imaging using genetically encoded sensors. J Vis Exp 2014;e51657 CrossRef Medline

62. Gruenwald K, Holland JT, Stromberg V, et al. Visualization of glutamine transporter activities in living cells using genetically encoded glutamine sensors. PLoS One 2012;7:e38591 CrossRef Medline

63. Xue M, Wei W, Su Y, et al. Supramolecular probes for assessing glutamine uptake enable semi-quantitative metabolic models in single cells. J Am Chem Soc 2016;138:3085-93 CrossRef Medline

64. Ghoreishi SM, Khalaj A, Sabzevari O, et al. Technetium-99m chelatorfree radiolabeling of specific glutamine tumor imaging nanoprobe: in vitro and in vivo evaluations. Int J Nanomedicine 2018;13:4671-83 CrossRef Medline

65. Jang K, Russo C, Di Ieva A. Radiomics in gliomas: clinical implications of computational modeling and fractal-based analysis. Neuroradiology 2020;62:771-90 CrossRef Medline 